\title{
Tuning the spin texture in binary and ternary surface alloys on $\operatorname{Ag}(111)$
}

\author{
Isabella Gierz, ${ }^{1, *}$ Fabian Meier, ${ }^{2,3}$ J. Hugo Dil, ${ }^{2,3}$ Klaus Kern, ${ }^{1,4}$ and Christian R. Ast ${ }^{1}$ \\ ${ }^{1}$ Max-Planck-Institut für Festkörperforschung, DE-70569 Stuttgart, Germany \\ ${ }^{2}$ Physik-Institut, Universität Zürich, CH-8057 Zürich, Switzerland \\ ${ }^{3}$ Swiss Light Source, Paul Scherrer Institute, CH-5232 Villigen, Switzerland \\ ${ }^{4}$ Institut de Physique de la Matière Condensée, Ecole Polytechnique Fédérale de Lausanne, CH-1015 Lausanne, Switzerland
}

(Received 8 November 2010; published 13 May 2011)

\begin{abstract}
A giant spin splitting has been observed in surface alloys on noble metal (111) surfaces as a result of a strong structural modification at the surface as well as the large atomic spin-orbit interaction (SOI) of the alloy atoms. These surface alloys are an ideal playground to manipulate both the size of the spin splitting as well as the position of the Fermi level, as it is possible to change the atomic SOI as well as the relaxation by varying alloy atoms and substrates. Using (spin- and) angular-resolved photoemission spectroscopy in combination with quantitative low-energy electron diffraction, we have studied the mixed binary $\mathrm{Bi}_{x} \mathrm{Sb}_{1-x} / \mathrm{Ag}(111)$ surface alloy where we observed a continuous evolution of the band structure with $x$ and the mixed ternary $\mathrm{Bi}_{0.3} \mathrm{~Pb}_{0.35} \mathrm{Sb}_{0.35} / \mathrm{Ag}(111)$ surface alloy.
\end{abstract}

DOI: 10.1103/PhysRevB.83.195122

PACS number(s): 73.20.At

\section{INTRODUCTION}

Spin degeneracy is the consequence of both time reversal and spatial inversion symmetry. The latter is broken at interfaces (surfaces) so that the two-dimensional (2D) states localized at this interface (surface) become spin polarized in the presence of spin-orbit interaction (SOI) as described by the Rashba-Bychkov (RB) model. ${ }^{1}$ The possibility to tune the spin splitting by an external gate voltage forms the basis for many spintronic device proposals such as the Datta-Das spin field effect transistor. ${ }^{2}$ Furthermore, a gradient in the effective magnetic field caused by a spatial variation of the Rashba-type spin splitting leads to spin separation in the Stern-Gerlachtype spin filter. ${ }^{3}$ In addition, these $2 \mathrm{D}$ spin-polarized states are expected to show the intrinsic spin Hall effect, ${ }^{4,5}$ and an enhancement of the superconducting transition temperature is predicted in the regime where the Rashba splitting is larger than the Fermi energy. ${ }^{6}$

The RB model also gives a qualitative description for spinpolarized states localized at the surfaces of heavy metals such as $\mathrm{Au}(111),{ }^{7-9} \mathrm{~W}(110),{ }^{10} \mathrm{Bi}(111), \mathrm{Bi}(110), \mathrm{Bi}(100),{ }^{11}$ and $\mathrm{Sb}(111) .{ }^{12}$ Recently, surface alloys, where every third atom in a noble metal (111) surface [Ag(111) (Refs. 13-19) or Cu(111) (Refs. 17 and 20)] is replaced by a heavy alloy atom $(\mathrm{Bi}, \mathrm{Pb}$, and $\mathrm{Sb}$ ), were identified as a new class of materials that exhibits a spin splitting that is orders of magnitude larger than, e.g., in semiconductor heterostructures. ${ }^{14}$

For future device applications, it is necessary to tune the size of the spin splitting as well as the position of the Fermi level in such Rashba systems so that the Fermi surface possesses the desired spin texture. A first step in this direction was the experimental proof that the spin splitting (and at the same time the Fermi energy) can be controlled via the Bi content in a mixed $\mathrm{Bi}_{x} \mathrm{~Pb}_{1-x} / \mathrm{Ag}(111)$ surface alloy. ${ }^{18,21}$

Here, we show by (spin- and) angular-resolved photoemisson spectroscopy [(S)ARPES] and quantitative low-energy electron diffraction $[I(V)$-LEED] that it is possible to form a well-ordered mixed binary $\mathrm{Bi}_{x} \mathrm{Sb}_{1-x} / \mathrm{Ag}(111)$ surface alloy where the spin splitting can be tuned, while leaving the position of the Fermi level largely unaffected. This is an important step toward the realization of a mixed ternary $\mathrm{Bi}_{x} \mathrm{~Pb}_{y} \mathrm{Sb}_{1-x-y} / \mathrm{Ag}(111)$ surface alloy, where spin splitting and Fermi energy can be tuned independently by varying the material parameters $x$ and $y$. In order to prove the possibility to grow sufficiently well-ordered ternary surface alloys, we present ARPES results for the ternary $\mathrm{Bi}_{0.3} \mathrm{~Pb}_{0.35} \mathrm{Sb}_{0.35} / \mathrm{Ag}(111)$ surface alloy and determine the size of the spin splitting by SARPES.

\section{A. The Rashba-Bychkov model}

The RB model, although applicable to qualitatively describe the spin splitting in many different 2D electron systems, was originally developed to explain the observed beating pattern in the magnetic susceptibility for $\mathrm{GaAs}_{\mathrm{Al}} \mathrm{Al}_{x} \mathrm{Ga}_{1-x} \mathrm{As}$ heterojunctions. ${ }^{1}$ The $2 \mathrm{D}$ electron gas that forms due to the band bending at the interface of such a semiconductor heterojunction is confined by an asymmetric potential that breaks space inversion symmetry. The electrons move with a certain velocity $\boldsymbol{v}$ (or in-plane momentum $\boldsymbol{k}_{\|}$) within the electric field $\boldsymbol{E}$ given by the gradient of the asymmetric confinement potential $\nabla V$. In the rest frame of the electron, this electric field is transformed into a magnetic field $\boldsymbol{B} \propto$ $\boldsymbol{k}_{\|} \times \nabla V$ that aligns the electron spin. The corresponding interaction Hamiltonian is given by $H=-\boldsymbol{\mu} \boldsymbol{B}$, where the magnetic moment of the electron $\boldsymbol{\mu}$ is proportional to the electron spin, i.e., to the Pauli matrices $\sigma$. This leads to the so-called Rashba-Bychkov Hamiltonian $H_{\mathrm{RB}}=\alpha_{R} \boldsymbol{\sigma}\left(\boldsymbol{k}_{\|} \times \boldsymbol{e}_{z}\right)$, where the Rashba constant $\alpha_{R}$ is proportional to the magnitude of the potential gradient $\nabla V$ and $\boldsymbol{e}_{z}$ is a unit vector that indicates the direction of $\nabla V$ perpendicular to the confinement plane of the $2 \mathrm{D}$ electron gas. ${ }^{1}$ Solving the Schrödinger equation within the framework of the nearly free electron (NFE) model leads to the following dispersion: $E\left(k_{\|}\right)=\frac{\hbar^{2}}{2 m^{*}}\left(k_{\|} \pm k_{R}\right)^{2}+$ $E_{0}$, where $m^{*}$ is the effective mass and $E_{0}$ is the position of the band maximum. This is illustrated in Fig. 1(a), where the momentum offset $k_{R}$ and the Rashba energy $E_{R}$ of the RB-type dispersion are indicated by arrows. The Rashba parameter $\alpha_{R}=\hbar^{2} k_{R} / m^{*}$ is a measure for the size of the spin splitting and closely related to the Rashba energy $E_{R}=\hbar^{2} k_{R}^{2} / 2 m^{*}$. 


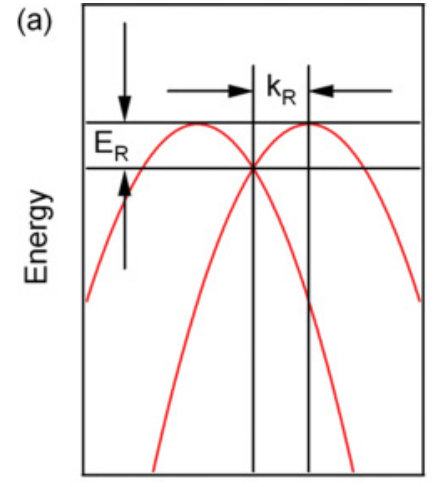

(b)

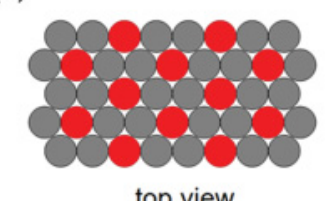

top view

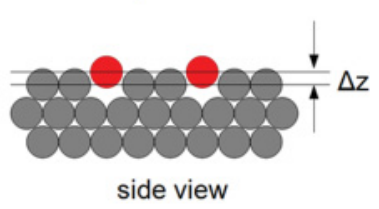

Wave Vector $\mathrm{k}$

FIG. 1. (Color online) (a) Characteristic dispersion of the 2D quasi-free-electron gas with RB-type spin-orbit interaction. (b) Surface alloys on noble metal (111) surfaces form a $(\sqrt{3} \times \sqrt{3}) R 30^{\circ}$ reconstruction where every third substrate atom (gray) in the topmost layer is replaced by an alloy atom (red, dark gray). The alloy atoms relax outward by an amount $\Delta z$.

The two parabolas are completely spin polarized, with the spin orientation perpendicular to both $\boldsymbol{k}_{\|}$and $\nabla V$. As $\nabla V$ lies along the surface normal in the framework of the RB model, the spin polarization is completely in plane and parallel to the circular constant energy contours.

The RB model was also successfully applied to qualitatively describe the spin-split dispersion on different noble metal surfaces such as, e.g., the surface state on $\mathrm{Au}(111) .{ }^{7}$ However, an estimation of the Rashba constant $\alpha_{R}$ based on the magnitude of the potential gradient at the surface in the NFE model is orders of magnitude too small. ${ }^{22,23}$ This problem is solved by a simple nearest-neighbor tight-binding model where the inversion asymmetry perpendicular to the confinement plane is mimicked by a nonzero overlap between $p_{x, y}$ and $p_{z}$ orbitals on neighboring atoms given by the asymmetry parameter $\gamma{ }^{22}$ In this case, the Rashba constant $\alpha_{R}$ was found to be proportional to the product of the atomic spin-orbit coupling strength $\alpha$ and the asymmetry parameter $\gamma$. Recent firstprinciples calculations investigated the microscopic origin of the RB-type spin splitting at surfaces. ${ }^{24}$ It was found that the potential gradient at the crystal surface leads to an asymmetric charge distribution in the vicinity of the nuclei, which can be characterized by the mixing of $l$ and $(l \pm 1)$ components in the surface-state wave function. Furthermore, the authors come to a conclusion similar to the one in Ref. 22 in the sense that the essential contribution to the RB-type spin splitting comes from regions close to the atomic cores.

\section{B. Surface alloys on $\operatorname{Ag}(111)$}

Surface alloys such as $(\mathrm{Bi}, \mathrm{Pb}, \mathrm{Sb}) / \mathrm{Ag}(111)$ exhibit a giant spin splitting that is orders of magnitude larger than the spin splitting of the pristine $\mathrm{Ag}(111)$ surface. The most obvious reason for the considerable increase in spin splitting upon formation of the surface alloy is the fact that every third atom of the $\operatorname{Ag}(111)$ surface is replaced by a heavy alloy atom $(\mathrm{Bi}, \mathrm{Pb}, \mathrm{Sb})$, which increases the atomic contribution to the spin splitting $\alpha$. Furthermore, in contrast to the clean (111) substrate, the surface alloy is corrugated due to the outward relaxation $\Delta z$ of the alloy atoms [see Fig. 1(b)]. The influence of this outward relaxation on the size of the spin splitting has been addressed by first-principles calculations, which predicted that an increasing outward relaxation of the $\mathrm{Bi}$ atoms in the $\mathrm{Bi} / \mathrm{Ag}(111)$ surface alloy increases the $p_{x, y}$ contribution to the otherwise $s p_{z}$ surface state and the spin splitting. ${ }^{25}$ These findings have been confirmed experimentally in Ref. 26.

The connection between $p_{x, y}$ admixture to the surface state due to the outward relaxation of the alloy atom and an increased spin splitting can be understood as follows. Calculations based on the NFE model have shown that an additional in-plane asymmetry of the potential increases the size of the spin splitting considerably. ${ }^{27}$ In the case of the surface alloys on $\operatorname{Ag}(111)$, this in-plane inversion asymmetry is given by the threefold rotational symmetry of the $\operatorname{Ag}(111)$ substrate. Upon increasing the outward relaxation of the alloy atom, the $p_{x, y}$ component of the surface-state wave function increases. As $p_{x, y}$ orbitals lie in the plane of the surface, they are particularly sensitive to the in-plane inversion asymmetry caused by the $\mathrm{Ag}(111)$ substrate, which results in a considerable enhancement of the spin splitting in surface alloys.

The characteristic parameters for the lower $s p_{z}$ band for the three surface alloys $(\mathrm{Bi}, \mathrm{Pb}, \mathrm{Sb}) / \mathrm{Ag}(111)$ are summarized in Table I. The Rashba constant $\alpha_{R}$ increases from $\mathrm{Sb}$ via $\mathrm{Pb}$ to $\mathrm{Bi}$ with increasing mass and increasing outward relaxation $\Delta z$ of the alloy atom. As $\mathrm{Bi}$ and $\mathrm{Sb}$ have the same number of valence electrons, i.e., they are isoelectronic, the band maximum $E_{0}$ is located at a similar energetic position in the occupied states for the $\mathrm{Bi} / \mathrm{Ag}(111)$ and the $\mathrm{Sb} / \mathrm{Ag}(111)$ surface alloys. $\mathrm{Pb}$, however, has one valence electron less than both $\mathrm{Bi}$ and $\mathrm{Sb}$. Therefore, the band maximum of the $s p_{z}$ state is in the unoccupied states. All the $s p_{z}$ states have negative effective masses ranging from $-0.10 m_{e}$ for $\mathrm{Sb} / \mathrm{Ag}(111)$ to $-0.35 m_{e}$ for $\mathrm{Bi} / \mathrm{Ag}(111)$, where $m_{e}$ is the free electron mass.

Mixing $\mathrm{Bi}$ and $\mathrm{Pb}$ in a binary $\mathrm{Bi}_{x} \mathrm{~Pb}_{1-x} / \mathrm{Ag}(111)$ surface alloy leads to a continuous evolution of the characteristic parameters of the $s p_{z}$ surface-state dispersion with Bi content

TABLE I. Characteristic parameters for the different surface alloys on $\operatorname{Ag}(111)$ and the $\operatorname{Ag}(111)$ substrate.

\begin{tabular}{lllccccc}
\hline \hline & \multicolumn{1}{c}{$\alpha_{R}$} & \multicolumn{1}{c}{$k_{R}$} & $E_{0}$ & $m^{*}$ & $\alpha$ & $\Delta z$ & Reference \\
\hline $\mathrm{Bi} / \mathrm{Ag}(111)$ & $3.2 \mathrm{eV} \AA$ & $0.13 \AA^{-1}$ & $-0.135 \mathrm{eV}$ & $-0.31 m_{e}$ & $1.25 \mathrm{eV}$ & $0.65 \AA$ & $14,15,26,28$ \\
$\mathrm{~Pb} / \mathrm{Ag}(111)$ & $1.52 \mathrm{eV} \AA$ & $0.03 \AA^{-1}$ & $+0.654 \mathrm{eV}$ & $-0.15 m_{e}$ & $0.91 \mathrm{eV}$ & $0.46 \AA$ & $13,15,26,28$ \\
$\mathrm{Sb} / \mathrm{Ag}(111)$ & $0.38 \mathrm{eV} \AA$ & $0.005 \AA^{-1}$ & $-0.28 \mathrm{eV}$ & $-0.10 m_{e}$ & $0.40 \mathrm{eV}$ & $0.10 \AA$ & $16,19,26,28$ \\
$\mathrm{Ag}(111)$ & $0.013 \mathrm{eV} \AA$ & $0.0007 \AA^{-1}$ & $-0.063 \mathrm{eV}$ & $+0.40 m_{e}$ & $0.11 \mathrm{eV}$ & $0.00 \AA$ & $8,24,29$ \\
\hline \hline
\end{tabular}


$x .^{18,21}$ The spin splitting $k_{R}$ increases with increasing $\mathrm{Bi}$ content accompanied by a downward shift of the band maximum into the occupied states. $\mathrm{Bi}$ and $\mathrm{Sb}$, on the other hand, are isoelectronic, i.e., mixing $\mathrm{Bi}$ and $\mathrm{Sb}$ in a binary $\mathrm{Bi}_{x} \mathrm{Sb}_{1-x} / \mathrm{Ag}(111)$ surface alloy would offer the possibility to tune the size of the spin splitting without changing the Fermi level. However, the $\mathrm{Sb} / \mathrm{Ag}(111)$ surface alloy forms with either face-centered-cubic (fcc) or hexagonally closed-packed (hcp) top-layer stacking depending on the growth conditions, ${ }^{26,30,31}$ whereas $\mathrm{Bi} / \mathrm{Ag}(111)$ as well as $\mathrm{Pb} / \mathrm{Ag}(111)$ always form with fcc top-layer stacking. Therefore, it is not a priori clear whether a well-ordered $\mathrm{Bi}_{x} \mathrm{Sb}_{1-x} / \mathrm{Ag}(111)$ surface alloy can be formed.

\section{EXPERIMENTS}

\section{A. Mixed binary $\mathrm{Bi}_{x} \mathrm{Sb}_{1-x} / \mathrm{Ag}(111)$ surface alloy}

All experiments were performed in ultrahigh vacuum with a base pressure of $1 \times 10^{-10}$ mbar. The ARPES experiments were done with a SPECS Phoibos 150 hemispherical analyzer with an energy resolution of $10 \mathrm{meV}$ and monochromatized He I radiation at $h v=21.2 \mathrm{eV}$. For the LEED measurements, an ErLEED 1000-A was used. ARPES and LEED experiments were performed at liquid-nitrogen temperature.

The spin splitting of the $s p_{z}$ surface state in $\mathrm{Sb} / \mathrm{Ag}(111)$ is smaller than the linewidth of the bands, which prevents its investigation by conventional spin-integrated ARPES. ${ }^{16}$ The discrimination of the two bands, however, can be achieved by spin-resolved ARPES (SARPES), where the spin polarization $P$ of the photoelectrons is measured in addition to their kinetic energy and the emission angle. ${ }^{32}$ Due to the low efficiency of present Mott detectors, SARPES measurements are very time consuming and are therefore usually restricted to single spin-resolved momentum distribution curves (MDCs). The intensities for spin-up (spin-down) electrons $I^{\uparrow}\left(I^{\downarrow}\right)$ are obtained from the measured spin-integrated intensity $I_{\text {tot }}$ according to $I^{\uparrow}=(1+P) I_{\text {tot }} / 2$ and $I^{\downarrow}=(1-P) I_{\text {tot }} / 2$. Assuming a parabolic dispersion, the Rashba splitting $k_{R}$ is then given by $k_{R}=\Delta k / 2$, where $\Delta k$ is the $\boldsymbol{k}_{\|}$distance of the maxima in $I^{\uparrow}$ and $I^{\downarrow}$. Note that $k_{R} \neq \Delta k / 2$ if the dispersion of the bands is not completely parabolic.

SARPES experiments were performed at the Surface and Interface spectroscopy beamline at the Swiss Light Source of the Paul Scherrer Institute using the COPHEE spectrometer. ${ }^{33}$ This spectrometer is equipped with two orthogonal Mott polarimeters, which can measure the spin expectation value for an arbitrary state in reciprocal space. The energy and angular resolution are $80 \mathrm{meV}$ and $4^{\circ}$, respectively. The data were obtained using synchrotron radiation of $24 \mathrm{eV}$ at room temperature.

The $\operatorname{Ag}(111)$ substrate was cleaned using several sputtering-annealing cycles (sputtering with $1 \mathrm{keV}$ Ar ions at an Ar pressure of $1 \times 10^{-6}$ mbar followed by annealing at $530{ }^{\circ} \mathrm{C}$ ). Cleanliness was controlled with $\mathrm{x}$-ray photoemission spectroscopy (XPS). In addition, the surface state of clean $\mathrm{Ag}(111)$ was monitored with ARPES. For the preparation of the mixed $\mathrm{Bi}_{x} \mathrm{Sb}_{1-x} / \mathrm{Ag}(111)$ surface alloy for the ARPES experiments, $\mathrm{Sb}$ and $\mathrm{Bi}$ were successively deposited on the $\operatorname{Ag}(111)$ substrate using a commercial electron beam evaporator. The substrate temperature was $250{ }^{\circ} \mathrm{C}$ during $\mathrm{Sb}$ deposition. For the subsequent Bi deposition, the substrate temperature was reduced to $150{ }^{\circ} \mathrm{C}$. For the SARPES experiments, $\mathrm{Bi}$ and $\mathrm{Sb}$ were deposited simultaneously. After deposition, the LEED pattern showed a sharp $(\sqrt{3} \times \sqrt{3}) R 30^{\circ}$ structure.

To prepare the ternary surface alloy $\mathrm{Bi}_{x} \mathrm{~Pb}_{y} \mathrm{Sb}_{1-x-y} / \mathrm{Ag}(111), \quad \mathrm{Sb}$ was deposited first with the sample at $200^{\circ} \mathrm{C}$ and then $\mathrm{Bi}$ and $\mathrm{Pb}$ were simultaneously deposited at $150{ }^{\circ} \mathrm{C}$. The LEED pattern of the ternary surface alloy showed a spot broadening, which we attribute to a larger disorder.

The $\mathrm{Bi}, \mathrm{Sb}$, and $\mathrm{Pb}$ coverage was determined by analyzing the integrated intensity of the respective core levels, measured with XPS.

\section{RESULTS AND DISCUSSION}

Figure 2(a) shows the evolution of the surface-state band structure for the mixed binary alloys $\mathrm{Bi}_{x} \mathrm{Sb}_{1-x} / \mathrm{Ag}(111)$ as a function of $\mathrm{Bi}$ content $x$ measured with ARPES. The dispersion of the $s p_{z}$ surface state evolves continuously between $x=0$ and 1 . Around $x=0.5$, the linewidth of the bands increases considerably, which we attribute mainly to disorder (details see below). The position of the lower $s p_{z}$ band was determined by fitting MDCs with a Lorentzian and a constant background. The resulting band dispersion $E(k)$ was then fitted by parabolas to determine the characteristic Rashba parameters. The continuous evolution of momentum offset $k_{R}$, Rashba constant $\alpha_{R}$, band maximum $E_{0}$, and effective mass $m^{\star}$ with increasing Bi content $x$ is shown in Fig. 2(b). The Rashba parameter $\alpha_{R}$ has been calculated from the experimentally determined momentum offset and effective mass.

The continuous evolution of $k_{R}, m^{\star}$, and $\alpha_{R}$ as a function of Bi content $x$ is in good agreement with recent first-principles calculations. ${ }^{34}$ It is well known that the magnitude of the potential gradient $\nabla V$ at the surface cannot account for experimentally observed spin splittings. Furthermore, we do not expect significant changes of the surface potential gradient $\nabla V$ with $\mathrm{Bi}$ content $x$. Therefore, we attribute the increase of the Rashba constant $\alpha_{R}$ upon increasing $\mathrm{Bi}$ content $x$ to the simultaneous increase in atomic SOI and outward relaxation $\Delta z$. In order to model mixed binary and ternary surface alloys, one may assume that the chemical and structural differences between the different alloy atoms can be taken into account by creating an artificial alloy atom, the properties (atomic spin-orbit interaction $\alpha$ and outward relaxation $\Delta \mathrm{z}$ ) of which are a linear interpolation between the properties of $\mathrm{Bi}, \mathrm{Pb}$, and $\mathrm{Sb}$, taken as a concentration-weighted average. ${ }^{34}$ The excellent agreement between these calculations and our experimental results is an indication that this approach is a valid approximation for the description of mixed surface alloys, although, in real samples, the alloy atoms are expected to keep their individual properties.

While $k_{R}, m^{\star}$, and $\alpha_{R}$ continuously increase with $x$, the band maximum $E_{0}$ reaches a maximum at about $x \approx 0.63$ and then decreases again. Figure 3(a) shows a more detailed analysis of the shift of the band maximum as a function of $\mathrm{Bi}$ content $x$. Intuitively, one might expect the band maximum to shift linearly with Bi content $x$. This is indicated by the black dashed line in Fig. 3(a). On top of this linear increase, we find 

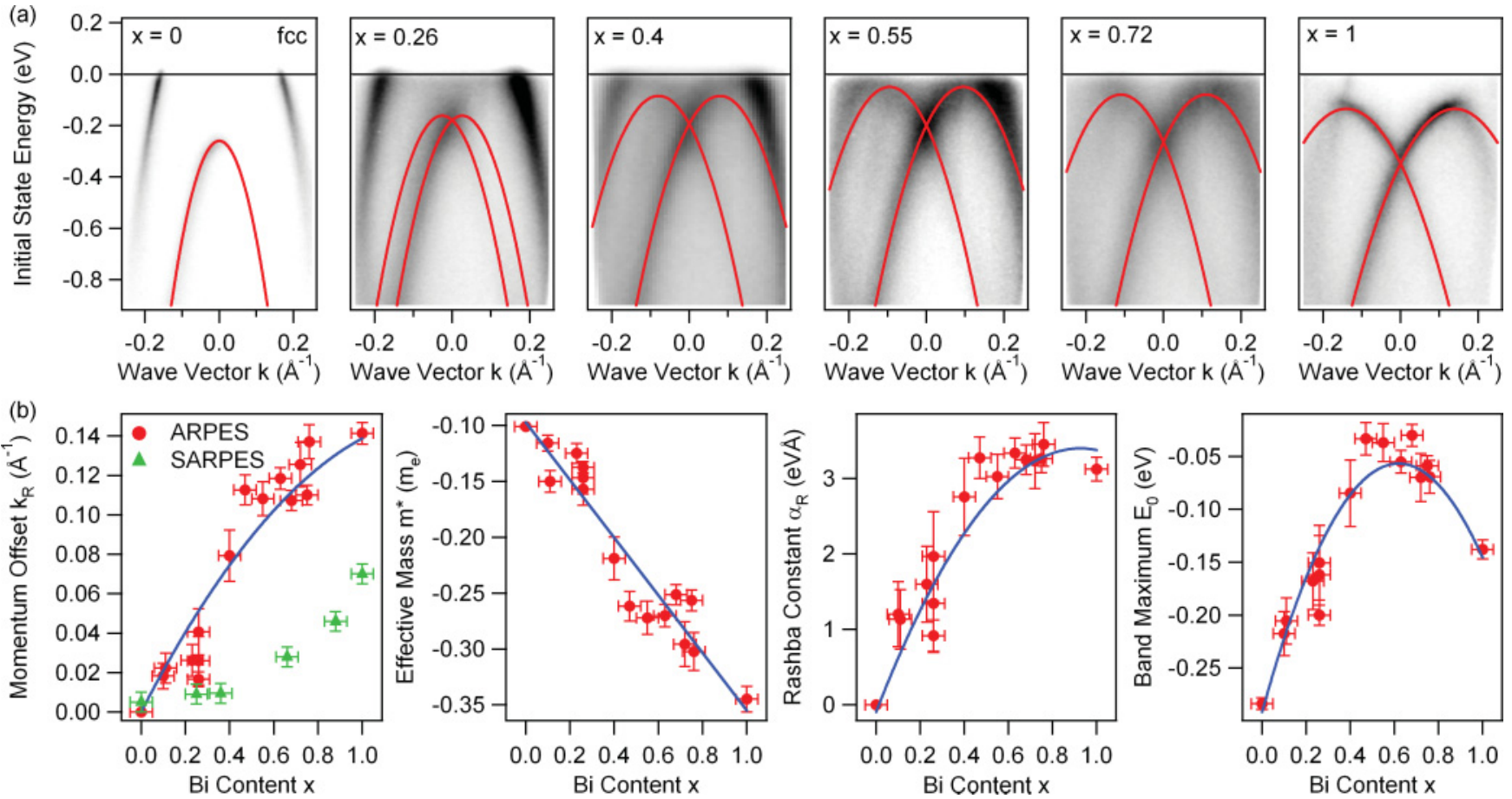

FIG. 2. (Color online) Experimental photoemission from $\mathrm{Bi}_{x} \mathrm{Sb}_{1-x} / \mathrm{Ag}(111)$. The evolution of the $s p_{z}$ surface-state dispersion is shown as a function of Bi content $x$ on a linear gray scale with black (white) corresponding to high (low) photocurrents (a). From parabolic fits to the data [solid lines in (a)], the characteristic parameters of the Rashba model (momentum offset $k_{R}$, effective mass $m^{\star}$, Rashba constant $\alpha_{R}$, and band maximum $E_{0}$ ) have been determined (b). Red dots and green triangles were obtained by spin-integrated and spin-resolved ARPES, respectively. Solid lines are guides to the eye.

a parabolic contribution [continuous black line in Fig. 3(a)] with a maximum at $x \approx 0.5$. The sum of the linear and the parabolic contribution results in a good fit of the experimental data points [gray line in Fig. 3(a)]. Note that the maximum of this fit is situated at $x=0.63$ instead of $x=0.5$.
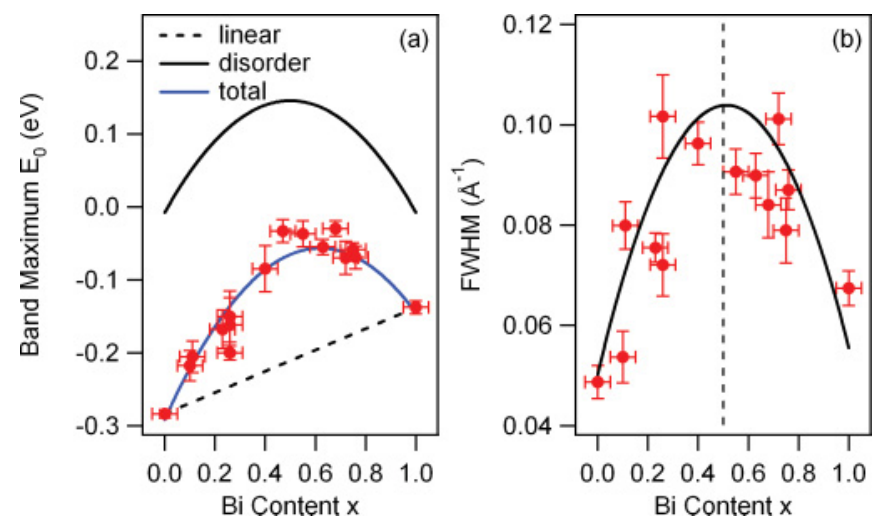

FIG. 3. (Color online) Panel (a) presents a more detailed analysis of the behavior of the band maximum $E_{0}$ as a function of Bi content $x$. On top of the expected linear increase (black dashed line), we find a parabolic contribution (continuous black line) with a maximum at $x \approx 0.5$. The blue (gray) line represents the sum of linear and parabolic contribution and nicely fits the experimental data points (red dots). Panel (b) shows the full width at half maximum (FWHM) of the $s p_{z}$ band at an initial state energy of $-0.8 \mathrm{eV}$ as a function of $x$. Both the parabolic contribution to position of $E_{0}$ as well as the linewidth exhibit a clear maximum around $x=0.5$.
Interestingly, the parabolic contribution to the shift of the band maximum $E_{0}$ correlates well with the behavior of the full width at half maximum (FWHM) of the $s p_{z}$ band. Figure 3(b) shows the FWHM of the $s p_{z}$ band at $E-E_{F}=$ $-0.8 \mathrm{eV}$ as a function of $\mathrm{Bi}$ content $x$, clearly revealing a maximum of the linewidth at $x \approx 0.5$. It is known that $E_{0}$ depends on the outward relaxation of the $\mathrm{Bi}$ and $\mathrm{Sb}$ atoms. ${ }^{25}$ However, it is unlikely that the outward relaxation of the alloy atoms in the mixed $\mathrm{Bi}_{x} \mathrm{Sb}_{1-x} / \mathrm{Ag}(111)$ surface alloy is larger than in the pure $\mathrm{Bi} / \mathrm{Ag}(111)$ surface alloy. As both the parabolic contribution to the band maximum $E_{0}$ as well as the linewidth show a maximum at $x \approx 0.5$, we attribute the maximum of $E_{0}$ to disorder.

From the experiments presented in this paper, it is difficult to deduce the exact nature of this disorder. One possible candidate is chemical disorder caused by the mixing of different alloy atoms. A second possibility is structural disorder due to the difference in outward relaxation between $\mathrm{Bi}$ and $\mathrm{Sb}$ atoms. For a better understanding, further investigations with, e.g., scanning tunneling microscopy are necessary, which go beyond the scope of this paper.

For small Bi contents $x$, the spin splitting of the $s p_{z}$ surface state of $\mathrm{Bi}_{x} \mathrm{Sb}_{1-x} / \mathrm{Ag}(111)$ is comparable to the linewidth of the bands. This limits the accuracy of the values for the spin splitting obtained by conventional spin-integrated ARPES. To overcome this problem, Fig. 4 shows spin-resolved MDCs recorded at an initial-state energy of $-0.6 \mathrm{eV}$ as a function of Bi content $x$ for the mixed $\mathrm{Bi}_{x} \mathrm{Sb}_{1-x} / \mathrm{Ag}(111)$ surface alloy. The SARPES-derived momentum offset $\Delta k / 2$ is included in Fig. 2(b) (green triangles). 

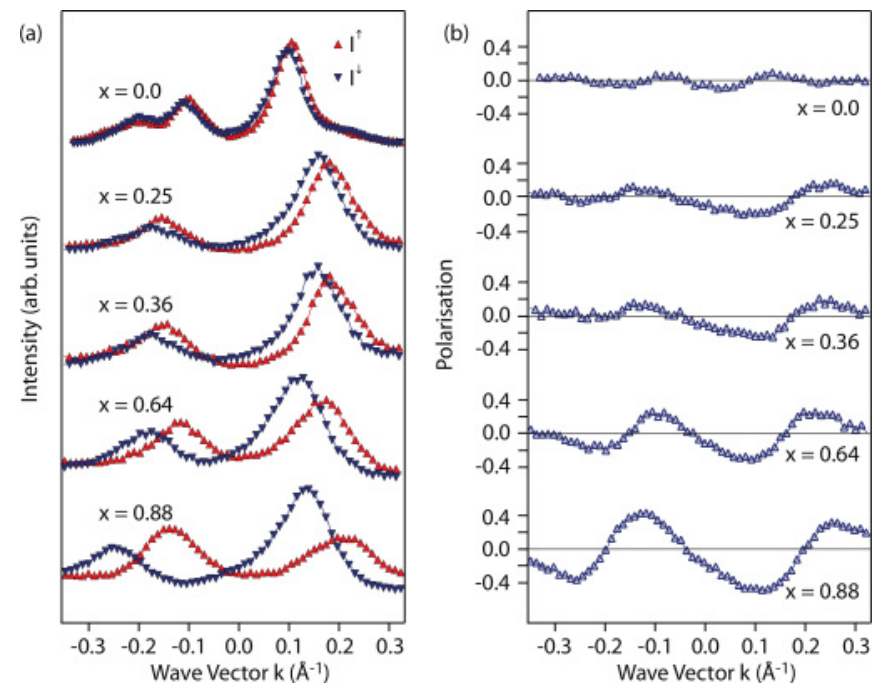

FIG. 4. (Color online) Spin-resolved photoemission from the mixed $\mathrm{Bi}_{x} \mathrm{Sb}_{1-x} / \mathrm{Ag}(111)$ surface alloy. Intensities $I^{\uparrow}$ (red triangles pointing upward) and $I^{\downarrow}$ (blue triangles pointing downward) (a) and spin-polarization (b) of momentum distribution curves from $\mathrm{Bi}_{x} \mathrm{Sb}_{1-x} / \mathrm{Ag}(111)$ at an initial-state energy of $-0.6 \mathrm{eV}$ as a function of Bi content $x$.

For a Bi content $x \gtrsim 0.3$, the $s p_{z}$ band is not parabolic any longer because it hybridizes with the upper $p_{x y}$ band. In this case, the values for $k_{R}$ as determined by ARPES and $\Delta k / 2$ as determined by SARPES deviate systematically. Nevertheless, the discrepancy for low values of $x$, where the dispersion is expected to be parabolic, is unexpectedly large. Although we cannot fully explain this discrepancy, we want to point out that, for small $x$, a determination of $k_{R}$ with conventional spinintegrated ARPES is difficult for several reasons. First, the size of the spin splitting is comparable to the linewidth of the bands, so the two spin-polarized parabolas cannot be properly resolved. Second, the photoemission intensity of the $s p_{z}$ state for small $x$ is suppressed for $k>0$ at $h v=21.2 \mathrm{eV}$ [see Fig. 2(a)] due to photoemission matrix element effects, so only part of the bands are available for the fitting procedure described above, which limits the accuracy of the fits. However, the main trend, a continuous increase of the spin splitting with Bi content $x$, is clearly reproduced by the SARPES data.

A more elaborate analysis of the spin-resolved data allows us to determine all three components of the photoelectron spin polarization as explained in Ref. 35. For small $x$, when the spin splitting is comparable to the momentum broadening, a spinstate interference in the photoemission process is observed in the region where states with orthogonal spinors overlap. This effect creates an artificial out-of-plane spin polarization that does not reflect the spin polarization of the initial state. ${ }^{19}$ However, this spin-state interference only takes place for small $x$ and can be well separated from the spin polarization resulting from the Rashba effect. We find that, in contrast to the size of the spin splitting, the spin direction of the initial state does not depend significantly on $x$, in agreement with previous results. ${ }^{18}$

The $(\sqrt{3} \times \sqrt{3}) R 30^{\circ}$ phase of $\mathrm{Sb} / \mathrm{Ag}(111)$ can be formed with either fcc or hcp top-layer stacking. ${ }^{26,30,31}$ The faulted hcp top-layer stacking of the $\mathrm{Sb} / \mathrm{Ag}(111)$ surface alloy is

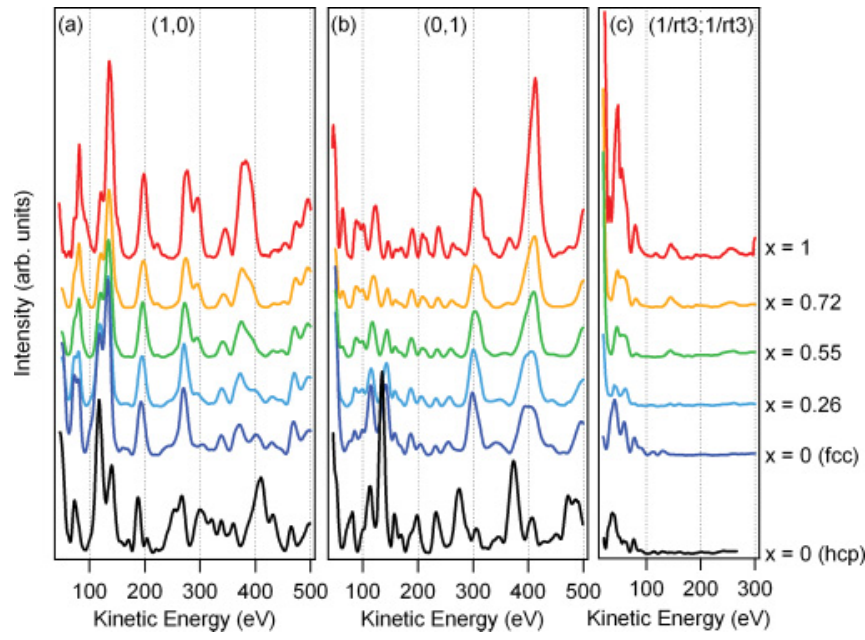

FIG. 5. (Color online) Low-energy electron diffraction from mixed $\mathrm{Bi}_{x} \mathrm{Sb}_{1-x} / \mathrm{Ag}(111)$ surface alloys. The $I(V)$ spectra represent the integrated intensities of the (a) $(1,0)$, (b) $(0,1)$, and (c) $(1 / \sqrt{3}$, $1 / \sqrt{3})$ spots versus electron energy. The spectra evolve continuously between $x=0$ and 1 . To exclude a possible hcp top-layer stacking, spectra for hcp-stacked $\mathrm{Sb} / \mathrm{Ag}(111)$ are displayed for comparison (bottom).

accompanied by the presence of subsurface stacking faults in the $\mathrm{Ag}$ substrate caused by $\mathrm{Sb}$ diffusion into the bulk. ${ }^{30} \mathrm{In}$ experiment, the top-layer stacking can be controlled by tuning the energy of the deposited $\mathrm{Sb}$ ions during $\mathrm{Sb}$ deposition with an electron beam evaporator. The $\mathrm{Sb} / \mathrm{Ag}(111)$ surface alloys with fcc and hcp top-layer stacking, respectively, can easily be identified with the help of $I(V)$-LEED measurements, where the intensity $I$ of a particular diffraction spot is measured as a function of the kinetic energy of the incident electrons, which is controlled by the acceleration voltage $V .{ }^{26}$

In order to determine the top-layer stacking for the mixed $\mathrm{Bi}_{x} \mathrm{Sb}_{1-x} / \mathrm{Ag}(111)$ surface alloy, we investigated the surface structure with $I(V)$-LEED (Fig. 5). The $I(V)$-LEED spectra were averaged over equivalent spots and smoothed (further details are given in Ref. 26). They evolve continuously between the pure $\mathrm{Bi} / \mathrm{Ag}(111)$ surface alloy (spectra for $\mathrm{x}=1$ in Fig. 5) and the pure $\mathrm{Sb} / \mathrm{Ag}(111)$ surface alloy with fcc top-layer stacking [spectra for $\mathrm{x}=0(\mathrm{fcc})]$. For comparison, the $I(V)$ spectra for the $\mathrm{Sb} / \mathrm{Ag}(111)$ surface alloy with hcp top-layer stacking are also shown [spectra for $\mathrm{x}=0$ (hcp)]. As these spectra clearly differ from those for the mixed $\mathrm{Bi}_{x} \mathrm{Sb}_{1-x} / \mathrm{Ag}(111)$ surface alloy, we conclude that it forms with fcc top-layer stacking even if $\mathrm{Sb}$ ions are deposited at sufficiently high energies to form an hcp-stacked surface alloy.

\section{A. Ternary surface alloy $\mathrm{Bi}_{x} \mathrm{~Pb}_{y} \mathrm{Sb}_{1-x-y} / \operatorname{Ag}(111)$}

As was shown before, both $\mathrm{Bi}_{x} \mathrm{~Pb}_{1-x} / \mathrm{Ag}(111)$ and $\mathrm{Bi}_{x} \mathrm{Sb}_{1-x} / \mathrm{Ag}(111)$ can be formed and exhibit a well-defined band structure. While in the $\mathrm{Bi}_{x} \mathrm{~Pb}_{1-x} / \mathrm{Ag}(111)$ surface alloy, both the spin splitting and the Fermi level change as a function of $x$, it is possible to change the spin splitting while leaving the Fermi level largely unaffected in the corresponding $\mathrm{Bi}_{x} \mathrm{Sb}_{1-x} / \mathrm{Ag}(111)$ surface alloy. As transport properties are determined by the spin texture of the Fermi surface, it is important to tune both the position of the Fermi level as 

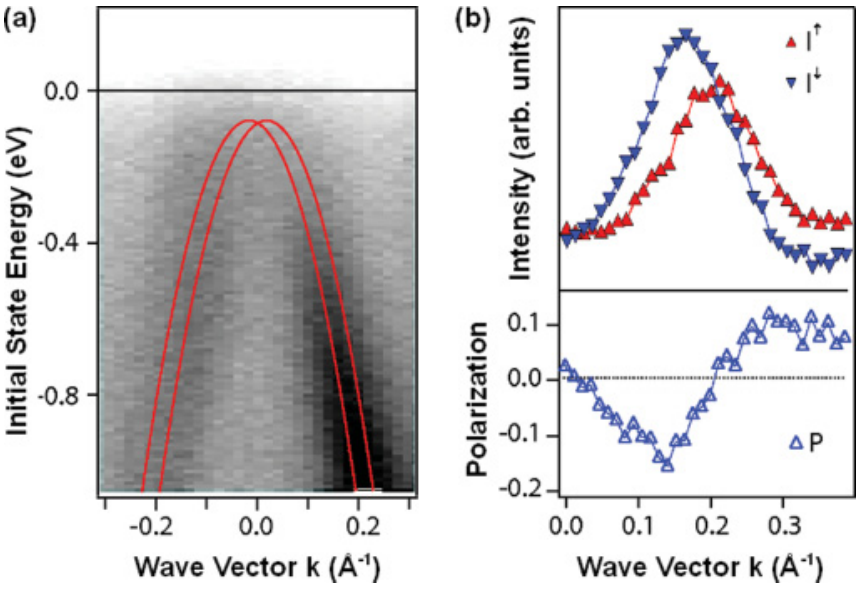

FIG. 6. (Color online) Spin-resolved photoemission from the ternary $\mathrm{Bi}_{0.3} \mathrm{~Pb}_{0.35} \mathrm{Sb}_{0.35} / \mathrm{Ag}(111)$ surface alloy. (a) Surface-state dispersion around $\bar{\Gamma}$. Solid lines are guides to the eye. The difference in intensity distribution across the band structure as compared to Fig. 2(a) is related to the different geometries of the two ARPES setups. (b) Spin-resolved intensities $I^{\uparrow}$ and $I^{\downarrow}$ and spin polarization $P$ measured at an initial-state energy of $-0.75 \mathrm{eV}$. The spin splitting of the $s p_{z}$ surface state is clearly resolved.

well as the size of the spin splitting independently. According to theoretical predictions, this can be achieved in a ternary $\mathrm{Bi}_{x} \mathrm{~Pb}_{y} \mathrm{Sb}_{1-x-y} / \mathrm{Ag}(111)$ surface alloy as follows. ${ }^{34}$ In order to increase $k_{R}$, one has to add heavy elements with large outward relaxation (i.e., $\mathrm{Bi}, \mathrm{Pb}$ ). For a modification of $E_{F}, \mathrm{Bi}_{x} \mathrm{Sb}_{1-x}$ should be mixed with $\mathrm{Pb}$.

Figure 6(a) shows the surface-state band structure for a mixed ternary surface alloy with $(x, y, z=1-x-y)$ $=(0.3,0.35,0.35)$. The linewidth is significantly increased as compared to those for the mixed binary surface alloys. The ARPES measurements in Fig. 6(a) were done using the
COPHEE spectrometer with a reduced angular resolution as compared to the setup used for the ARPES data in Fig. 2. An additional increase in linewidth is caused by the intermixing of three elements (instead of two) and by a (probably) not optimized sample preparation. Despite the large linewidth, the spin splitting $\left(\Delta k / 2=0.019 \AA^{-1}\right)$ can still be clearly resolved with SARPES, as shown in Fig. 6(b).

\section{CONCLUSIONS}

We have shown that it is possible to form the mixed binary $\mathrm{Bi}_{x} \mathrm{Sb}_{1-x} / \mathrm{Ag}(111)$ surface alloy despite the fcc and hcp top-layer stacking of $\mathrm{Sb} / \mathrm{Ag}(111)$. Furthermore, $I(V)$ LEED experiments revealed that the mixed $\mathrm{Bi}_{x} \mathrm{Sb}_{1-x} / \mathrm{Ag}(111)$ surface alloy always forms with fcc top-layer stacking. ARPES and SARPES measurements show a continuous evolution of the band structure with $\mathrm{Bi}$ content $x$. The results on the mixed ternary surface alloy $\mathrm{Bi}_{0.3} \mathrm{~Pb}_{0.35} \mathrm{Sb}_{0.35} / \mathrm{Ag}(111)$ show that ternary alloys can be formed and exhibit a reasonably welldefined band structure. Our findings indicate the possibility to form ternary surface alloys with arbitrary compositions that allow us to tune the spin splitting as well as the Fermi energy independently and continuously over a broad range of values.

Following this idea, the situation where the Fermi level lies between the band maximum and the crossing point of the two parabolas is particularly interesting: in this regime, the spins on the two circular Fermi surfaces rotate in the same direction and the density of states shows quasi-one-dimensional behavior. ${ }^{15}$ In this case, the Rashba energy becomes the dominating energy scale and an increase of the transition temperature into a superconducting state is expected. ${ }^{6}$ Furthermore, mixed surface alloys with a spatial variation of their chemical composition $x$ create a gradient of the effective Rashba field that is a prerequisite for building a Stern-Gerlach spin filter. ${ }^{3}$
*Corresponding author: i.gierz@ fkf.mpg.de

${ }^{1}$ Y. A. Bychkov and E. I. Rashba, Pis'ma Zh. Eksp. Teor. Fiz. 39, 66 (1984) [JETP Lett. 39, 78 (1984)].

${ }^{2}$ S. Datta and B. Das, Appl. Phys. Lett. 56, 665 (1990).

${ }^{3}$ J. I. Ohe, M. Yamamoto, T. Ohtsuki, and J. Nitta, Phys. Rev. B 72, 041308(R) (2005).

${ }^{4}$ J. Sinova, D. Culcer, Q. Niu, N. A. Sinitsyn, T. Jungwirth, and A. H. MacDonald, Phys. Rev. Lett. 92, 126603 (2004).

${ }^{5} \mathrm{~J}$. Wunderlich, B. Kaestner, J. Sinova, and T. Jungwirth, Phys. Rev. Lett. 94, 047204 (2005).

${ }^{6}$ E. Cappelluti, C. Grimaldi, and F. Marsiglio, Phys. Rev. Lett. 98, 167002 (2007).

${ }^{7}$ S. LaShell, B. A. McDougall, and E. Jensen, Phys. Rev. Lett. 77, 3419 (1996).

${ }^{8}$ F. Reinert, G. Nicolay, S. Schmidt, D. Ehm, and S. Hüfner, Phys. Rev. B 63, 115415 (2001).

${ }^{9}$ J. Henk, A. Ernst, and P. Bruno, Phys. Rev. B 68, 165416 (2003).

${ }^{10}$ E. Rotenberg, J. W. Chung, and S. D. Kevan, Phys. Rev. Lett. 82, 4066 (1999).

${ }^{11}$ Y. M. Koroteev, G. Bihlmayer, J. E. Gayone, E. V. Chulkov, S. Blügel, P. M. Echenique, and P. Hofmann, Phys. Rev. Lett. 93, 046403 (2004).
${ }^{12}$ K. Sugawara, T. Sato, S. Souma, T. Takahashi, M. Arai, and T. Sasaki, Phys. Rev. Lett. 96, 046411 (2006).

${ }^{13}$ D. Pacilé, C. R. Ast, M. Papagno, C. Da Silva, L. Moreschini, M. Falub, A. P. Seitsonen, and M. Grioni, Phys. Rev. B 73, 245429 (2006).

${ }^{14}$ C. R. Ast, J. Henk, A. Ernst, L. Moreschini, M. C. Falub, D. Pacilé, P. Bruno, K. Kern, and M. Grioni, Phys. Rev. Lett. 98, 186807 (2007).

${ }^{15}$ C. R. Ast, G. Wittich, P. Wahl, R. Vogelgesang, D. Pacilé, M. C. Falub, L. Moreschini, M. Papagno, M. Grioni, and K. Kern, Phys. Rev. B 75, 201401(R) (2007).

${ }^{16}$ L. Moreschini, A. Bendounan, I. Gierz, C. R. Ast, H. Mirhosseini, H. Höchst, K. Kern, J. Henk, A. Ernst, S. Ostanin, F. Reinert, and M. Grioni, Phys. Rev. B 79, 075424 (2009).

${ }^{17}$ H. Bentmann, F. Forster, G. Bihlmayer, E. V. Chulkov, L. Moreschini, M. Grioni, and F. Reinert, Europhys. Lett. 87, 37003 (2009).

${ }^{18}$ F. Meier, V. Petrov, S. Guerrero, C. Mudry, L. Patthey, J. Osterwalder, and J. H. Dil, Phys. Rev. B 79, 241408 (2009).

${ }^{19}$ F. Meier, V. Petrov, H. Mirhosseini, L. Patthey, J. Henk, J. Osterwalder, and J. H. Dil, J. Phys. Condens. Matter 23, 072207 (2011). 
${ }^{20}$ L. Moreschini, A. Bendounan, H. Bentmann, M. Assig, K. Kern, F. Reinert, J. Henk, C. R. Ast, and M. Grioni, Phys. Rev. B 80, 035438 (2009).

${ }^{21}$ C. R. Ast, D. Pacilé, L. Moreschini, M. C. Falub, M. Papagno, K. Kern, M. Grioni, J. Henk, A. Ernst, S. Ostanin, and P. Bruno, Phys. Rev. B 77, 081407 (2008).

${ }^{22}$ L. Petersen and P. Hedegård, Surf. Sci. 459, 49 (2000).

${ }^{23} \mathrm{R}$. Winkler, in Spin-Orbit Coupling Effects in Two-Dimensional Electron and Hole Systems, edited by J. Kühn, Th. Müller, A. Ruckenstein, F. Steiner, J. Trümper, and P. Wölfle (Springer, Berlin, 2003).

${ }^{24}$ G. Bihlmayer, Y. M. Koroteev, P. M. Echenique, E. V. Chulkov, and S. Blügel,Surf. Sci. 600, 3888 (2006).

${ }^{25}$ G. Bihlmayer, S. Blügel, and E. V. Chulkov, Phys. Rev. B 75, 195414 (2007).

${ }^{26}$ I. Gierz, B. Stadtmüller, J. Vuorinen, M. Lindroos, F. Meier, J. H. Dil, K. Kern, and C. R. Ast, Phys. Rev. B 81, 245430 (2010).
${ }^{27}$ J. Premper, M. Trautmann, J. Henk, and P. Bruno, Phys. Rev. B 76, 073310 (2007).

${ }^{28}$ K. Wittel and R. Manne, Theor. Chim. Acta 33, 347 (1974).

${ }^{29}$ C. E. Moore, Atomic Energy Levels, National Bureau of Standards (US), Circ. No. 467 (US GPO, Washington, D. C., 1949, 1952), Vols. II and III.

${ }^{30}$ D. P. Woodruff and J. Robinson, J. Phys. Condens. Matter 12, 7699 (2000).

${ }^{31}$ P. D. Quinn, D. Brown, D. P. Woodruff, P. Bailey, and T. C. Q. Noakes, Surf. Sci. 511, 43 (2002).

${ }^{32}$ J. H. Dil, J. Phys. Condens. Matter 21, 403001 (2009).

${ }^{33}$ M. Hoesch, T. Greber, V. N. Petrov, M. Muntwiler, M. Hengsberger, W. Auwärter, and J. Osterwalder, J. Electron Spectrosc. Relat. Phenom. 124, 263 (2002).

${ }^{34}$ H. Mirhosseini, A. Ernst, S. Ostanin, and J. Henk, J. Phys. Condens. Matter 22, 385501 (2010).

${ }^{35}$ F. Meier, H. Dil, J. Lobo-Checa, L. Patthey, and J. Osterwalder, Phys. Rev. B 77, 165431 (2008). 